\title{
Raltegravir Use in Special Populations
}

\author{
Margaret Johnson \\ HIV/AIDS Services, Royal Free Hospital NHS Trust, London, U.K.
}

\begin{abstract}
Raltegravir, the first approved integrase inhibitor, has been shown to be virologically effective in Phase II and Phase III clinical trials in both treatment naïve and triple class resistant patients. It also has an excellent tolerability profile and lacks significant drug-drug interactions making it an important drug in the treatment of a number of special patient populations. In this review its use in patients undergoing solid organ and bone marrow transplantation and patients receiving cancer chemotherapy, will be discussed. In addition other indications including patients with metabolic complications of existing antiretroviral drugs as well as patients with side effects on current HAART regimens. Other groups of patients where raltegravir may play an important role are patients with renal disease and tuberculosis. Finally, although not licensed for use in pregnancy, raltegravir may need to be considered in some pregnant women with antiretroviral resistance or tolerability issues with current HAART regimens.
\end{abstract}

\section{INTRODUCTION}

Raltegravir, the first approved intergrase inhibitor, has been shown to be virologically effective in phase II and III clinical trials in both treatment naïve $[1,2]$ and triple class resistant $[3,4]$ patients. These studies have shown that raltegravir is well tolerated and has no effect on lipids and has the advantage that in in-vitro studies it has significantly less drug interactions than other antiretroviral agents, make it an important addition to our treatment armenterium for patients with HIV infection.

In vitro studies show that raltegravir is not a substrate of cytochrome P450 (CYP) enzymes and does not inhibit CYP1A2, CYP2B6, CYP2C8, CYP2C9, CYP2C19, CYP2D6 or CYP3A. It does not induce CYP3A4 and does not inhibit P-glycoprotein-mediated transport. Based on these data raltegravir is not expected to effect the pharmacokinetics of drugs that are substrates of these enzymes or P glycoprotein. In vivo and in vitro studies demonstrate raltegravir is eliminated mainly by metabolism via a UGTIAI-mediated glucuronidation pathway. Raltegravir is an important option for patients with comorbidities and needing medications which interact with antiretroviral agents.

The above attributes of efficacy, the excellent tolerability profile and the lack of significant drug-drug in- teractions make raltegravir an attractive option for many patients with HIV infection. It may play an important role in patients undergoing solid organ and bone marrow transplantation, patients receiving chemotherapy for malignant disease and some patients with HIV/TB co-infection. Other groups of patients with HIV infection may also benefit from raltegravir. This will include patients with dyslipidaemia and patients who currently have side-effects from antiretroviral therapy in particular the boosted PI's. In addition, in this review, the use of raltegravir in pregnancy will be discussed. A further possible future role for raltegravir may be in post exposure prophylaxis where its mode of action and good tolerability could make an attractive component of future PEP regimens.

\section{RALTEGRAVIR UsE IN SOLID ORgAN TRANSPLANTATION}

Solid organ liver and renal transplantations are now performed successfully in selected HIV infected patients. However, multiple and reciprocal drug-drug interactions are observed between antiretroviral drugs and the calcinerin inhibitors through CYP450 metabolisation. Raltegravir is not a substrate of CYP450 enzymes, as discussed above, while the nonnucleoside reverse transcriptase inhibitors (NNRTI's) and particularly boosted protease inhibitors $\mathrm{PI} / \mathrm{r}$ have significant drug drug interactions with the calcinerin inhibitors, ciclosporin and tacrolimus. The avoidance of $\mathrm{PI} / \mathrm{r}$ based therapy post transplantation may lead to more manageable interactions and better transplant outcomes. There has already been some experience from investigators in the UK, Italy and France of the use of raltegravir in combination with 2 nucleoside reverse transcriptase inhibitors (NRTI's) for both patients undergoing liver transplantation and renal transplantation with sustained virological response (11 months of follow up) and no apparent interaction with immunosuppressant therapy leading to good graft function $[5,6,7]$.

The advantage of using raltegravir, a drug of high antiviral potency and non-significant interactions with immunosuppressants make this a possible safe and effective choice of agent in the management of these patients. Standard doses of raltegravir can be used with ciclosporin, tacrilimus and mycophenolate, assuming no other drug reactions. Its use reduces the need for dose adjustments, simplifying patient management. 


\section{RaLtegravir use in Bone Marrow TRANSPLANTATION}

As for those patients undergoing liver and renal transplantation, ciclosporin is used in bone marrow transplantation which is now being used in patients with HIV related lymphomas and other non-HIV related haematological malignancies. Again, the use of ciclosporin can cause difficulty in using this with current HAART regimens. Raltegravir may again be a very useful alternative for those patients avoiding the use of boosted PIs and NNRTI's for the period of ciclosporine use. Patients who develop graft versus host disease may need to be on ciclosporin for very long prolonged periods of time and for these patients in particular, raltegravir is a very important option.

\section{Raltegravir Use in Patients Receiving CHEMOTHERAPY}

Several recent cohort studies have shown that nonAIDS-defining cancers are more common in HIV-infected patients than HIV in uninfected persons $[8,9]$. The chemotherapeutic agents used in the treatment of many malignancies are often metabolised by CYP isoenzymes. There is however very little information particularly for for older chemotherapeutic drugs on drug interactions with any CYP inhibitors or inducers and very little data of drug-drug interactions with the NNRTI's and PI/r. Potentially, the use of NNRTIs and PI/HAART may lead to increased toxicity. In patients with non Hodgkin's lymphoma there is evidence that $\mathrm{PI} / \mathrm{r}$ based HAART leads to profound and prolonged neutropenia [10].

Many patients receiving chemotherapy will acquire fungal prophylaxis and again drug - drug interactions of anti-fungals and antiretroviral agents can result in complicated drug interactions. The drug of choice in non vincristine based chemotherapeutic regimens is itraconazole, however NRTI based HAART will lead to almost undetectable itraconazole levels and increased risk of fungal infection. Fluconazole is an alternative but does not prevent aspergillus infection which limits its use. Ambisone is also a possible option but it is very expensive and carries the increased risk of renal complications. The use of raltegravir is part of HAART regimens for these patients provides an attractive alternative option.

\section{Raltegravir Use in Patients with HiV/TB CO-INFECTION}

Patients with TB are usually treated with efavirenz based HAART and continue on standard doses of rifampicin [11]. However there will be a group of patients for whom efavirenz is inappropriate because of transmitted or acquired NNRTI resistance and also would not be the drug of choice in patients with transmitted or acquired NRTI resistance. In this group of patients a raltegravir based regimen is an attractive option to allow the patient to continue rifampicin based TB treatment. There is at present no real data on the correct dose of raltegravir with rifampicin. The raltegravir data sheets suggests using raltegravir $800 \mathrm{mg}$ b.d. without any pharmacokinetic evidence. Many would argue that levels of raltegravir are not as important as with other antiretroviral agents and standard doses would be effective. Clearly, more data is needed [11].

In patients started on an efavirenz based regimen who have to switch therapy because of virological failure or tolerability reasons there will be a two week period when $\mathrm{PI} / \mathrm{r}$ based HAART would be compromised by enzyme induction of both rifampicin and efavirenz. There may again be a case for the use of raltegravir if however a PI/r regimen is preferred, raltegravir could be considered for 2 - 4 weeks while rifampicin is washed out and the PI/r regimen can be safely initiated.

\section{Raltegravir Use in Pregnancy}

Raltegravir is not licensed for use in pregnancy and to date there is minimal information of the use of this drug in pregnancy [12]. Pregnancy however should never preclude the use of an optimal antiretroviral therapy to treat the mother $[12,13]$. In addition it is critical to reduce the viral load to undetectable levels to reduce the risk of mother to child transmission.

Raltegravir is classified as an FDA pregnancy category $\mathrm{C}$ (Table 1) and there is no evidence of teratogenicity in animal studies. At present safety and pharmacokinetic data are insufficient to recommend its use during pregnancy but as discussed above therapies of known benefit to a woman should not be withheld during pregnancy unless there are known adverse

Table 1. FDA Pregnancy Categories.

\begin{tabular}{|c|c|}
\hline Category & Interpretation \\
\hline \multirow[t]{2}{*}{ A } & Controlled studies show no risk \\
\hline & $\begin{array}{l}\text { Adequate, well-controlled studies in pregnant } \\
\text { women have failed to demonstrate risk to the } \\
\text { foetus }\end{array}$ \\
\hline \multirow[t]{2}{*}{ B } & No evidence of risk in humans \\
\hline & $\begin{array}{l}\text { Either animal findings show risk (but human } \\
\text { findings do not) or, if no adequate studies have } \\
\text { been done, animal findings are negative. }\end{array}$ \\
\hline \multirow[t]{2}{*}{ C } & Risk cannot be ruled out \\
\hline & $\begin{array}{l}\text { Human studies are lacking and animal studies } \\
\text { are either positive for foetal risk or lacking as } \\
\text { well. However, potential benefits may justify the } \\
\text { potential risk. }\end{array}$ \\
\hline \multirow[t]{2}{*}{ D } & Positive evidence of risk \\
\hline & $\begin{array}{l}\text { Investigational or postmarking data show risk to } \\
\text { foetus. Nevertheless, potential benefits may out- } \\
\text { weight the risk. }\end{array}$ \\
\hline \multirow[t]{2}{*}{$\mathbf{X}$} & Contraindicated in pregnancy \\
\hline & $\begin{array}{l}\text { Studies in animals or humans, or investigational } \\
\text { or postmarketing reports have shown foetal risk } \\
\text { which clearly outweighs any possible benefit to } \\
\text { the patient. }\end{array}$ \\
\hline
\end{tabular}


effects on the mother, foetus or infant and these adverse effects outweigh the benefit to the woman. Raltegravir with its potent activity against multi-drug resistant HIV should be considered for patients with a drug resistance profile indicating the need for raltegravir as a component of a HAART regimen for the treatment of the womens HIV infection.

There are also women who are unable to tolerate $\mathrm{PI} / \mathrm{r}$ regimens during pregnancy because of side effects of nausea, vomiting and/or diarrhoea. The use of efavirenz is not advised during pregnancy even in the third trimester. Many women are not suitable for treatment with nevirapine because of liver impairment and/or low CD4 counts. In this situation raltegravir again could be considered as a possible agent in an antiretroviral regimen.

There are a small group of patients who present very late in pregnancy with high viral loads and raltegravir could be an important agent in the management of these patients because of its rapid virological suppression when compared to other antiretroviral agents. It is important for clinicians to ensure that they report all patients with pre-natal exposure to raltegravir to the antiretroviral pregnancy registry at www.APRregistry.com so that safety data is collected.

\section{Raltegravir Therapy Use in Patients With Metabolic Complications of HIV INFECTION}

With patients living longer with HIV infection and taking antiretroviral therapy in the long-term, we are seeing an increased risk of metabolic complications with an increase of coronary events, dyslipidemia, insulin resistance, diabetes and hepatic steatosis [14]. Although the mechanisms are not fully understood, they are clearly multifactorial in origin and may include uncontrolled HIV infection, intermittent HAART therapy, antiretroviral therapy, hepatitis $\mathrm{C}$ co-infection, and lifestyle issues. All of these factors appear important and contribute to increased cardiovascular risk in patients [15-17].

Lipid disorders are common in HIV patients even prior to starting antiretroviral therapy and when patients start therapy, the problem may exacerbate particularly in patients receiving PI/r. Hypertriglyceridemia is common in patients receiving kaletra and there are lipid alterations with low dose Ritonavir when used in combination with all the protease inhibitors with this being demonstrated both in HIV positive patients and HIV negative individuals. Atazanavir, darunavir and fosamprenavir can be administered with a lower dose of Ritonavir, $100 \mathrm{mg}$ a day and this results in fewer lipid effects than kaletra but abnormal lipids remain problematic in some patients.

NNRTI use in particular with the use of efavirenz is also not infrequently associated with an increase in total cholesterol and LDL cholesterol levels when compared with lipid profiles of treatment naïve individuals. This increase is counterbalanced by an increase in HDL cholesterol for patients on nevirapine regimens.

Dyslipidemia also occurs with the use of NRTIs particularly zidovudine, DDI, stavudine and recent data from the DAD cohort and SMART trials showed an in- creased relative risk of myocardial infarction for patients receiving abacavir and didanosine. The risk for abacavir has not yet been fully substantiated or explained.

Raltegravir in both its phase 2 and phase 3 clinical trials in treatment naïve patients has been shown to have no effect on lipids [1-4] and thus may be a very good choice for patients at high cardiovascular risk using established multivariate risk models such as the Framingham equation. All patients starting and changing HAART regimens should undergo a cardiovascular risk assessment before and those patients at increased cardiovascular risk, raltegravir should be considered as a possible option.

For existing patients on antiretroviral regimens with abnormal lipid profiles, switching the efavirenz or $\mathrm{PI} / \mathrm{r}$ to raltegravir has been proposed as a potential strategy. In the SWITCHMARK studies [18], two multi-centre double blind randomised studies were performed in patients with undetectable viral loads on combination antiretroviral therapy containing kaletra to evaluate the effect of switching from kaletra to raltegravir versus continuing on the same regimen. The primary endpoint included the mean percentage change in lipids at 12 weeks and the demonstration of non-inferiority with respect of viral efficacy. Preliminary results from the study demonstrated that changing to raltegravir was associated with improved lipid profiles, however the pre-defined margin for non-inferiority was not reached. Patients entering the study could have previously had virological failure and therefore may have nucleoside and non-nucleoside resistance and account for the lack of viral efficacy. Viral suppression of course must always be the priority but many clinicians would consider that as long as a patient had no baseline resistance when starting antiretroviral therapy, had had an undetectable viral load $(<50$ copies $/ \mathrm{ml})$ on their current antiretroviral regimen, and had never failed a previous HAART regimen then switching an NNRTI or PI/ $r$ to raltegravir to manage dyslipidemia would be a reasonable strategy.

\section{Raltegravir Use with Renal Disease}

Patients with renal disease often present to clinicians with challenges in selecting an appropriate regimen. Tenofovir which is now used in most first line treatment regimens cannot be used safely in some patients with renal disease even with reduced doses, alternative agents should be considered whenever possible. Abacavir/lamivudine is a possibility for those who are at low risk for cardiovascular disease and those who have a pre-treatment HIV RNA of $<100,000$ copies. However most patients with renal disease these also have high cardiovascular risk and the consideration of an NRTI sparing regimen to include raltegravir maybe considered although data on such regimens are as yet limited.

\section{Raltegravir Use in Patients with TOLERABILITY IsSUES ON NNRTI AND PI REGIMENS}

Raltegravir has to date been shown to have an excellent side effect profile and maybe a very useful option 
in patients with side effects on current HAART regimens. Some patients on PI/r regimens have substantial problems with gastrointestinal intolerance with nausea, vomiting and diarrhoea. In addition patients on efavirenz based regimens may have CNS or psychiatric side effects. In addition there will b e a further group of patients with toxicity to NRTIs. For these patients raltegravir should be considered as a possible component of a HAART regimen.

\section{Post Exposure Prophylaxis}

To date there is no data on the use of Raltegravir in post exposure prophylaxis. However data on current PEP regimens suggest that they are poorly tolerated with many patients both healthcare workers requiring PEP and individuals receiving antivirals post-sexual exposure stopping treatment before they have completed the course. Raltegravir should be investigated in PEP regimens because of its excellent tolerability profile. In addition the possible combination of a CCR 5 entry inhibitor with an intergrase inhibitor has been suggested as a possible option for future PEP regimens. Studies to investigate these new strategies are clearly needed.

\section{SUMMARY}

Raltegravir the first licensed intergrase inhibitor is a drug with excellent tolerability, has no effect on lipids and has the added advantage of not being a substrate cytochrome P450 and therefore results in a lack of drug-drug interactions.

It will prove to be a very useful agent in the treatment of many patients with HIV infection particularly the special populations discussed in this review. Data will continue to accrue which will further define its role in the management of patients with HIV infection.

\section{REFERENCES}

1. Markovitz M, et al. Rapid and durable antiretroviral therapy effect of in treatment-naïve patients with HIV infection: results of a 48 week controlled study. J Acquir Immune Defic Syndr 2007; 46:125.

2. Lennox J, Dejesus E, Lazzarin A, et al. Subgroup analyses from STARTMARK, a Phase III study of raltegravirbased versus efavirenz-based combination therapy in treatment-naïve HIV-infected patients. Presented at the 16th Annual Conference on Retroviruses and Opportunistic Infections, Montreal, Canada February 8-11th, 2009; abstract \# 573.

3. Grinsztejn B, Nguyan BY, Katlama C, et al. Safety and efficacy of the HIV-1 intergrase inhibitor raltegravir (MK0158) in treatment experienced patients with multidrugresistant virus: a phase II randomised controlled trial. Lancet 2007; 369:1261.

4. Grinsztejn B. 48 week efficacy and safety of MK-0158, a novel HIV-1 intergrase inhibitor in patients with triple class resistant virus. 47th Interscience Conference on Antimicrobial Agents and Chemotherapy, Chicago 2007 (Abstract \#H713).

5. Di Biagio I A. Lack of interaction between raltegravir and cyclosporine in an HIV-infected liver transplant recipient. Journal of Antimicrobial Chemotherapy 2009; 64(4):874875.
6. Tricot L. Safety and efficacy of raltegravir in HIV-infected transplant patients co-treated with immunosuppressive drugs. Am J Transplant. 2009 Aug; 9(8): 1946-52.

7. Moreno A. Safe use of raltegravir and sirolimus in an HIV-infected patient with renal impairment after orthotopic liver transplantation. AIDS 2008 Feb 19; 22(4): 547548.

8. Burgi A, Brodine S, Wenger S, et al. Incidence and risk factors for the occurrence of non-AIDS-defining cancers among human immunodeficiency virus-infected individuals. Cancer 2005; 104: 1505-1511.

9. Kirk GD, Merlo C, O'Driscoll P, et al. HIV infection is associated with an increased risk for lung cancer, independent of smoking. Clin Infect Dis 2007; 45: 103-110.

10. Bower M, McCall P, Peat N, Ryan N, et al. Protease inhibitors, potentiate Chemotherapy-induced neutropenia. Blood 2004; 104: 2943-2946.

11. Pozniak A L, Collins S, Coyne KM, et al. British HIV Association guidelines for the treatment of TB/HIV co-infection 2009 - HIV Medicine in press.

12. Public Health Service Task Force Recommendations for use of antiretroviral drugs in pregnant women for maternal health and interactions to reduce perinatal HIV transmission in the United States. www.AIDSinfo.nih.gov (Accessed July 29th 2008)

13. Antiretroviral Pregnancy Registry Steering Committee. Antiretroviral Pregnancy Registry International interim report for 1 Jan 1989 - 31 Jan 2008. Willmington, NC: Registry Co-ordinating Centre ; 2007.

Available at: http://www.APRegistry.com.

14. Barbaro G. Highly active antiretroviral therapy - associated metabolic syndrome: Pathogenesis and cardiovascular risk. Am J Ther 2006 ; 13: 248.

15. El Sadr WM, Lundgren JD, Neaton JD, et al. CD4 countguided interruption of antiretroviral treatment. N Eng J Med 2006; 355: 2283-2296.

16. Kristofferson US, Kronberg G, Giger AK. A longitudinal study of changes in circulating markers of endothelial function in HIV patients starting combination ART. 15th Conference on Retroviruses and Opportunistic Infections. Boston MA, Feb 2008 Abstract 953.

17. Friis-Moller N, Weber R, Reiss P, et al. Cardiovascular risk factors in HIV patients - association with antiretroviral therapy. Results from DAD Study. AIDS 2003; 17: 1179-1193.

18. Eron J, Andrade J, Zajdenverg R, et al. Switching from stable lopinavir/ritonavir based to raltegravir based combination ART resulted in a superior lipid profile at week 12 but did not demonstrate non-inferior virological efficacy at week 24. 16th Conference on Retroviruses and Opportunistic Infections, Feb 8-11, 2009: Montreal, Canada Abstract 70aLB.

Address for correspondence:

Professor Margaret Johnson, MD FRCP

Consultant Physician and Clinical Director

HIV/AIDS Services

Royal Free Hospital NHS Trust

Professor of HIV Medicine

University College,

London

E-mail: Margaret.Johnson@royalfree.nhs.uk 